\title{
Vision Problems in Mild Traumatic Brain Injury
}

\section{Kenneth J Ciuffreda*}

SUNY/State College of Optometry, 33 West 42nd Street, New York, NY 10036, USA

*Corresponding author: Kenneth J Ciuffreda, SUNY/State College of Optometry, 33 West 42nd Street, New York, NY 10036, USA, Tel: 212-938-5765; E-mail: kciuffreda@sunyopt.edu

Received date: November 10, 2016; Accepted date: November 21, 2016; Published date: November 28, 2016

Copyright: ( $\odot 2016$ Ciuffreda KJ. This is an open-access article distributed under the terms of the Creative Commons Attribution License, which permits unrestricted use, distribution, and reproduction in any medium, provided the original author and source are credited.

\section{Introduction}

One of the many areas that may be adversely affected in an individual following a mild traumatic brain injury (mTBI), as well as TBI in general is vision. This is not surprising, since the majority of the cranial nerves, as well as 30-40 areas of the brain, involve basic vision and visual information processing of a sensory, motor, and/or perceptual nature $[1,2]$.

Over the past 20 years, there have been great strides made to identify individuals who may have experienced an mTBI, as this can be difficult in many cases where there is a sparse case history and vague symptoms, both of a general and visual nature. Hence, there has been the advent of more accurate, precise, objective and quantitative clinical and laboratory approaches for the detection and diagnosis of mTBIrelated vision problems $[2,3]$. This has included:

1. Clinical assessment of saccadic eye movements for accuracy and completion time with a global tracking task; accuracy is impaired, and completion time is increased, in those with mTBI as compared to a visually-normal, matched control group;

2. Clinical assessment of the near point of convergence: this is receded in many patients having visually-based near symptoms;

3. Objectively-based measures of saccadic latency and peak saccadic velocity: latency is increased with eventual natural recovery over several days, and the peak velocity may initially be reduced, with eventual natural recovery within the first month after the insult;

4. Objectively-based measures of accommodative and vergence peak velocity: in many cases, these and related oculomotor parameters are reduced and may remain abnormal after the natural recovery period of 6-9 months;

5. Objectively-based measures of the pupillary light reflex peak and average velocity: in many patients, these and related parameters are reduced and may remain abnormal after the natural recovery period; furthermore, pupillary dysfunction has been proposed to be, at least in part, contributory to the common symptom of photosensitivity;

6. Objectively-based measures of the visually-evoked response (VEP) amplitude and implicit time: in many patients, the former is slightly reduced and the latter is increased substantially, especially with a very low-luminance stimulus;

7. Objectively-based measures of the visually-evoked response amplitude incorporating binasal occluders, in those with the symptom of visual motion sensitivity: with addition of the occluders, the response amplitude is increased when compared to the non-occluder test value.

However, once the diagnosis of a visual problem in the mTBI patient is made, what's next? Can it be remediated? The answer in many cases is a resounding yes, with long-term persistence of the positive effects. Again, over the past decade or so, similar to the above for improving diagnostic capability, there have been considerable investigations and advances into the area of vision remediation, including both case reports, case series, retrospective studies and clinical trials, some of which used objective techniques to assess the therapeutic effect $[2,3]$.

In many cases, it involved what has become referred to as "neurooptometric vision rehabilitation" (NOR), or more simply "vision rehabilitation", based on the sound scientific principles of 'perceptual and motor learning', and optics. NOR is frequently performed in conjunction with either an occupational therapist or vision therapist, and in many cases with referral from a neurologist, physiatrist, physical therapist and even a specialty lawyer, as well in others. NOR involves the use of several approaches, frequently concurrently for remediation of common vision symptoms in this population [1-6]. This has included:

1. Lenses to improve accommodation, as well as the neurological interaction between accommodation and vergence, to attain single, clear, comfortable, and sustained binocular vision at near;

2. Vergence prisms to improve fusional ability and overall binocular vision at all distances;

3. Yoked prisms to improve spatial localization and visuomotor activities at all distances;

4. Lens tints and coatings to reduce photosensitivity and glare;

5. Selective/full occlusion to block the offensive diplopic image in cases of acquired strabismus, as well as binasal occluders to reduce the amount of visual motion perceived in those with visual motion sensitivity;

6. Oculomotor-based vision therapy to improve eye tracking ability of the versional, accommodative and vergence systems, and their interactions.

In addition to the aforementioned successful clinical approaches, there have been a number of laboratory-based studies demonstrating efficacy of these approaches using a range of metrics, including:

1. Objective techniques involving quantitative assessment of saccadic eye movements/reading eye movements, accommodative static and dynamic aspects, and vergence static and dynamic aspects, all following a brief period $(9-10 \mathrm{~h})$ of oculomotor-based vision therapy/rehabilitation;

2. Objective techniques to assess cortically-based activity (VEP amplitude) following brief oculomotor-based vision therapy/ rehabilitation; 
Page 2 of 2

3. Objective techniques to assess cortically-based alpha activity related to general/visual attention following brief oculomotor-based vision therapy/rehabilitation;

4. Objective techniques to assess cortically-based activity (VEP amplitude) following incorporation of binasal occluders;

5. Improvement in clinical vision signs using a comprehensive optometric clinical examination protocol;

6. Reduction in clinical vision symptoms using a standard case history, as well as with a validated symptom questionnaire (CISS).

Lastly, what holds for the future in the area of vision-based correlates related to mTBI? There are several likely directions [2,7]. First, there will be development of instrumentation and related efficient/rapid protocols for detection of an mTBI, as well as assessing either its natural recovery or improvement following some therapeutic intervention. Second, and related to the first, there will be development of devices and protocols to assess for acute concussion/mTBI in both the sports side-lines and military theatre, which may include portable MRI and VEP systems. Third, there will be additional quantitative, objective approaches to assess brain function in the emergency room, with further advances in pupillometry. Fourth, there will be testing of the above ideas during each of the three stages of mTBI, namely the acute, sub-acute and chronic phases, as each phase appears to have somewhat different abnormal parameters, at least for the pupil and saccadic systems. Lastly, one of the most active areas is, and will continue to be, that of uncovering vision-based, "objective biomarkers" for mTBI per se, especially those involving a simple and non-invasive manner, such as dynamic pupillometry [7].

The future in this area looks bright for our patients with mTBI.

\section{References}

1. Suter PS, Harvey LH (2011) Vision rehabilitation. CRC Press. Boca Raton, FL.

2. Ciuffreda KJ, Ludlam DL, Yadav NY, Thiagarajan P (2016) Traumatic brain injury: Visual consequences, diagnosis and treatment. Advances in ophthalmology and optometry 1: 307-333.

3. Ciuffreda KJ (2016) Compendium of works on visual rehabilitation by Dr. Kennenth J. Ciuffreda. Optometric Extension Program Foundation, Timonium.

4. Suchoff IB, Ciuffreda KJ, Kapoor N (2001) Visual and vestibular consequences of acquired brain injury. Optometric Extension Program Foundation, Santa Ana, CA.

5. Ciuffreda KJ, Ludlam DP, Kapoor N (2009) Clinical oculomotor training in traumatic brain injury. Optom Vis Devel 40: 16-23.

6. http://www.COVD.org

7. Ciuffreda KJ, Ludlam DP, Thiagarajan P, Yadav NK, Capo-Aponte J (2014) Proposed objective visual system biomarkers for mild traumatic brain injury (mTBI). Mil Med 179: 1212-1217. 